ARTICLE

\title{
Universal growth of ultra-thin III-V semiconductor single crystals
}

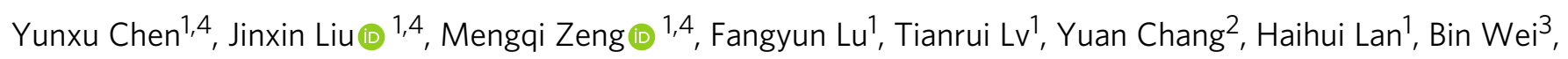
Rong Sun ${ }^{3}$, Junfeng $\mathrm{GaO}^{2}$, Zhongchang Wang $\mathbb{D}^{3}$ \& Lei Fu (iD) ${ }^{1 凶}$

Ultra-thin III-V semiconductors, which exhibit intriguing characteristics, such as twodimensional (2D) electron gas, enhanced electron-hole interaction strength, and strongly polarized light emission, have always been anticipated in future electronics. However, their inherent strong covalent bonding in three dimensions hinders the layer-by-layer exfoliation, and even worse, impedes the 2D anisotropic growth. The synthesis of desirable ultra-thin III-V semiconductors is hence still in its infancy. Here we report the growth of a majority of ultra-thin III-V single crystals, ranging from ultra-narrow to wide bandgap semiconductors, through enhancing the interfacial interaction between the III-V crystals and the growth substrates to proceed the 2D layer-by-layer growth mode. The resultant ultra-thin single crystals exhibit fascinating properties of phonon frequency variation, bandgap shift, and giant second harmonic generation. Our strategy can provide an inspiration for synthesizing unexpected ultra-thin non-layered systems and also drive exploration of III-V semiconductorbased electronics.

\footnotetext{
${ }^{1}$ College of Chemistry and Molecular Sciences, Wuhan University, Wuhan 430072, China. ${ }^{2}$ Laboratory of Materials Modification by Laser, Ion and Electron Beams, Dalian University of Technology, Ministry of Education, Dalian 116024, China. ${ }^{3}$ International Iberian Nanotechnology Laboratory (INL), Av. Mestre Jose Veiga s/n, 4715-330 Braga, Portugal. ${ }^{4}$ These authors contributed equally: Yunxu Chen, Jinxin Liu, Mengqi Zeng. ${ }^{凶}$ email: leifu@whu.edu.cn
} 
II-V semiconductors have profound underlying physics, such as high charge carrier mobility ${ }^{1}$, high electron drift velocity ${ }^{2}$, efficient light detection ${ }^{3}$ and emission ${ }^{4}$, which endow them great potential in high-speed, high-frequency electronics ${ }^{1}$ and high-efficiency optoelectronics including solar cells ${ }^{5}$, lasers ${ }^{6}$, and light-emitting diodes ${ }^{4,7}$. Once it comes to two dimensions, enormous unique characteristics emerge, such as twodimensional (2D) electron gas $^{8}$, enlarged lattice constant ${ }^{9,10}$, blue-shifted bandgap ${ }^{11,12}$, strong electron-hole interaction, and strongly polarized light emission favoring the nonlinear optics ${ }^{13}$, which stimulate the interest of their property exploration ${ }^{14}$ in the 2D limit.

However, the strong covalent bonding in the inherent zinc blende $(\mathrm{ZB})^{15,16}$ or wurtzite structures ${ }^{17}$ of these non-layered III-V crystals hinders the layer-by-layer exfoliation, and even worse, it greatly impedes the $2 \mathrm{D}$ anisotropic growth. Therefore, the synthesis of desirable ultra-thin III-V compound semiconductors is still in its infancy. Currently, among the various semiconductors crystals available, only ultra-thin GaN crystals have been synthesized ${ }^{11,12,18}$. The quantum confinement induced characteristics of a majority of these compounds, such as phosphides and antimonides, remain unproven in the $2 \mathrm{D}$ limit. The root cause is that these growth methods rely heavily on a nanoscale confined space $^{12}$ or a predesigned template $e^{11,18}$ with harsh requirements, thus seriously restricting the expansion of the growth methods to other ultra-thin III-V systems. Therefore, it is urgent to develop a general approach without strict presetting for the efficient growth of these ultra-thin III-V systems.

Here, alloy substrates with improved adhesion strength toward III-V crystals were designed to enhance the interfacial interaction between the substrates and the materials. Thus, the resulted surface energy difference can trigger the 2D layer-by-layer (LBL) growth behavior of III-V crystals on the substrates, further providing a driving force to stimulate the $2 \mathrm{D}$ anisotropic growth of various representative non-layered III-V single crystals ranging from ultra-narrow to wide bandgap semiconductors. These highly-crystalline ultra-thin single crystals exhibit intriguing properties of strong phonon confinement, apparent bandgap shift, and giant second harmonic generation (SHG). These assynthesized ultra-thin III-V systems can provide a fertile platform for probing their intrinsic physics. The as-proposed method offers a universal strategy for adjusting the growth mode of the materials and paves a route to achieve $2 \mathrm{D}$ crystals, which can bring forth inspiration for realizing the anisotropic growth of unexpected $2 \mathrm{D}$ materials, especially for the non-layered systems.

\section{Results}

Universality of the growth strategy. To create the surface energy differences, III-group-metal based alloys (e.g., $\mathrm{AuIn}_{2}$ ) with enhanced adhesion strength toward III-V crystals were employed as growth substrates. The strengthened interfacial interaction enables the growth of III-V crystals to proceed in the 2D LBL growth mode on the substrates, further leading to the formation of ultra-thin non-layered III-V single crystals, as sketched in Fig. 1a. However, in terms of the growth process performed on a pure III-group metal substrate with a weak adhesion strength (Fig. 1b), which leads to a three-dimensional (3D) island growth mode of the III-V crystals. An isotropic growth behavior would be triggered, thus resulting in the formation of bulk crystals.

Representative ultra-thin III-V single crystals were synthesized via the LBL growth strategy (Fig. 2a). Au is added to the In metal to form $\mathrm{AuIn}_{2}$ substrate and red $\mathrm{P}$ powders are employed as $\mathrm{V}$ group precursors during the chemical vapor deposition (CVD) process, leading to the growth of ultra-thin InP, as revealed in the scanning electron microscope (SEM) image (Fig. 2b). The typical
Raman spectrum of InP (Supplementary Fig. 1a) characterizes two peaks at $304 \mathrm{~cm}^{-1}$ and at $342 \mathrm{~cm}^{-1}$, which are assigned to the transverse optical (TO) mode and the longitudinal optical (LO) mode, respectively. The high intensity of the TO phonon frequency is deemed to be correlated with a polarized firstorder Raman scattering originated from the interplay of phonon confinement ${ }^{19}$. This spectral polarization confirms that the ultrathin InP crystals are of high quality. Compared with the Raman spectrum of the bulk InP where the two peaks are located at 306 and $345 \mathrm{~cm}^{-1}$ (Supplementary Fig. 2), the TO and LO phonon frequencies of ultra-thin InP exhibit distinguishable downshifts, which can be attributed to the size-induced phonon confinement effect $^{20,21}$ and the increased lattice constant ${ }^{22}$ owing to the reduced thickness.

We further show that varying the V-group precursors to $\mathrm{Sb}$ gives rise to the successful growth of ultra-thin InSb single crystals (Fig. 2c) on the AuIn 2 substrate. The TO $\left(177 \mathrm{~cm}^{-1}\right)$ and LO $\left(187 \mathrm{~cm}^{-1}\right)$ phonon frequencies (Supplementary Fig. 1b) of InSb single crystals exhibit similar peak-position-shift phenomena observed in the ultra-thin InP single crystals, which stems from the phonon confinement as well ${ }^{23}$. When varying the alloy substrate to be AuGa, ultra-thin GaP (Fig. 2d) and GaSb (Fig. 2e) single crystals can be synthesized. Compared with the Raman peaks of bulk $\mathrm{GaP}^{24}$ and $\mathrm{GaSb}^{23}$, the peaks of the ultra-thin $\mathrm{GaP}$ (Supplementary Fig. 1c) and GaSb (Supplementary Fig. 1d) also exhibit obvious downshifts. Apart from the narrow-bandgap III-V compounds mentioned above, the wide bandgap compound, such as ultra-thin GaN crystal (Fig. 2f), can also be synthesized on the AuGa substrates once the precursors are changed to urea $\left(\left(\mathrm{NH}_{2}\right)_{2} \mathrm{CO}\right)$ powders. The strong peak at 566 $\mathrm{cm}^{-1}\left(\mathrm{E}_{2}\right)$ suggests the high quality of ultra-thin $\mathrm{GaN}^{11}$. The growth of these representative III-V semiconductors provides a reliable evidence for the great universality of our strategy.

Theoretical analysis of the LBL growth process. The fundamental thermodynamic process of the growth was then explored for in-depth comprehending of how the InP growth modes were affected by the substrate with a strong adhesion strength. According to the classical thin film growth theory ${ }^{25}$, the growth modes of the material on a substrate can be defined by the difference of surface energies $(\Delta \gamma)$, which can be expressed as

$$
\Delta \gamma=\gamma_{m}+\gamma_{i}-\gamma_{s}
$$

where the $\gamma_{m}$ is the surface energy of the material, $\gamma_{s}$ is the surface energy of the substrate, and $\gamma_{i}$ is the interface energy between the material and the substrate. In addition, according to the Dupré equation

$$
\gamma_{i}=\gamma_{m}+\gamma_{s}-\beta_{i}
$$

where the $\beta_{i}$ is the adhesion energy between the material and the substrate, the $\Delta y$ can then be described as ${ }^{26}$

$$
\Delta \gamma=2 \gamma_{m}-\beta_{i}
$$

if $\Delta y<0$, the material growth can proceed in the $2 \mathrm{D} L B L$ growth mode on the substrate, which signifies the requirements of a high adhesion energy between the material and the substrate.

Given this, enhancing the interaction between the III-V compounds with the substrate can promote the LBL growth behavior and enable us to achieve the ultra-thin III-V single crystal. Here, the adhesion strengths of the growth substrates were adjusted via constructing appropriate III-metal-based alloy substrates. Taken the growth of ultra-thin InP as an example, we performed the density functional theory (DFT) calculations to investigate the interface adhesion energies of InP crystal layer on various substrate surfaces. As shown in Fig. 3a, the results suggest the adhesion energy of InP layer on the $\mathrm{AuIn}_{2}$ alloy is about 1.51 


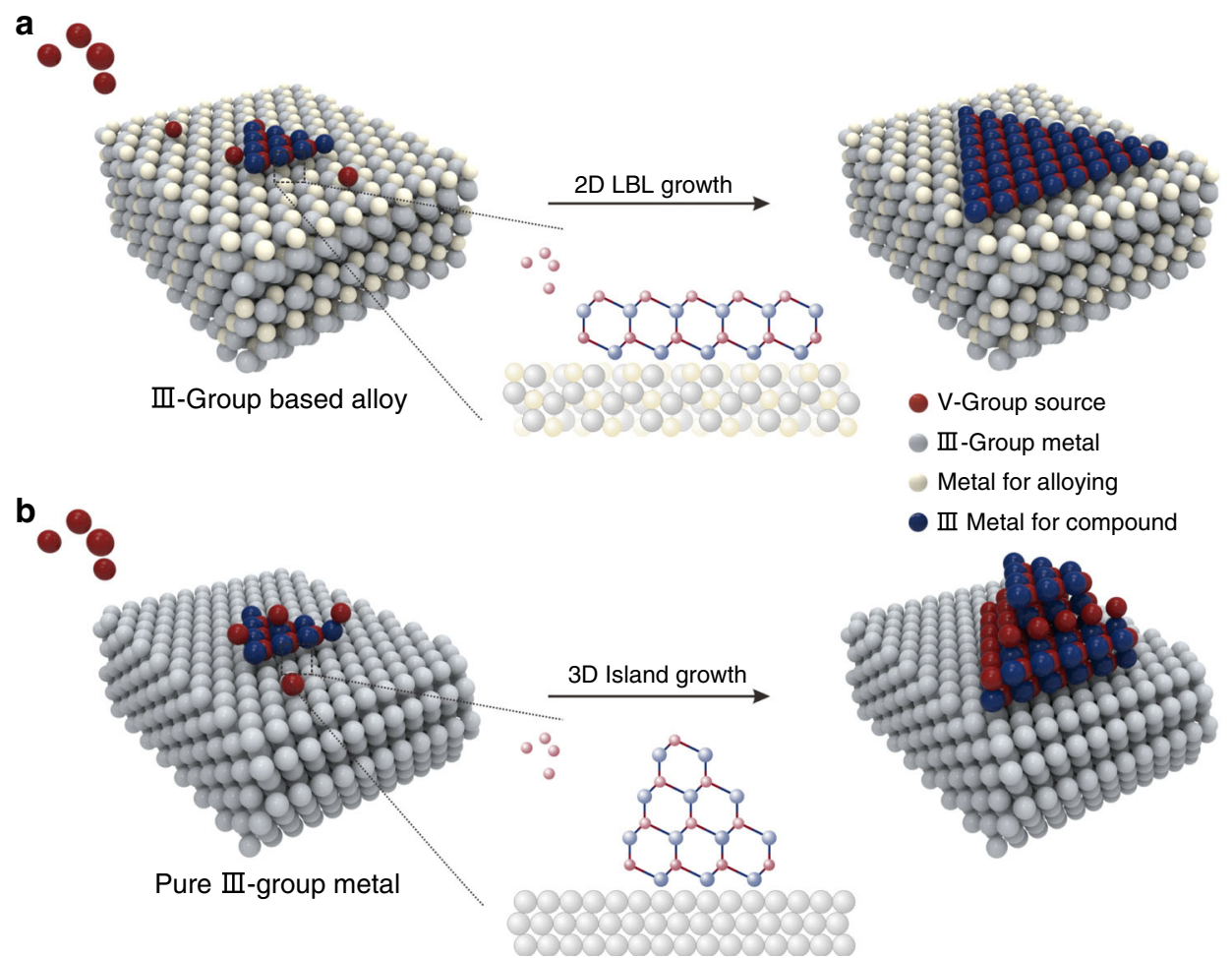

Fig. 1 Schematic illustration of the growth process of III-V crystals. a The schematic of the 2D LBL growth process of ultra-thin III-V single crystals on an alloy substrate. $\mathbf{b}$ The schematic of the 3D island growth process of bulk III-V single crystals on the pure III-group metal substrate.
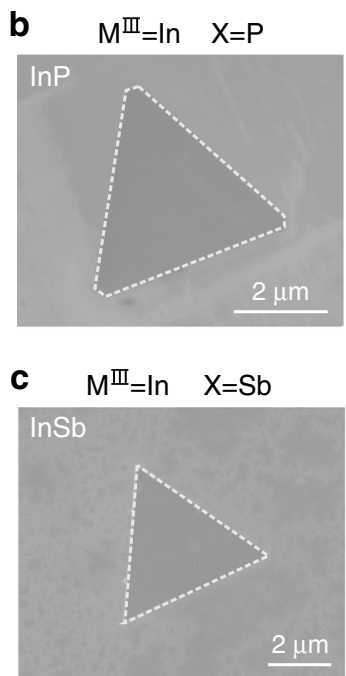

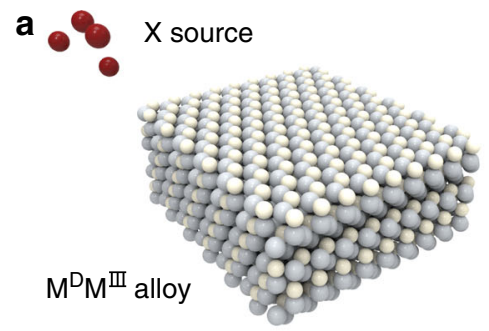

d

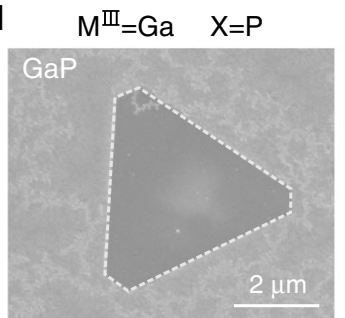

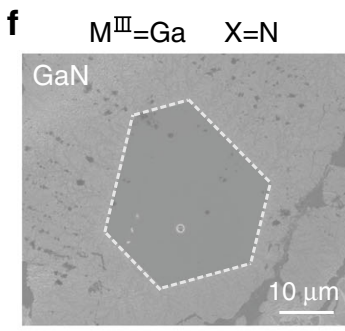

e

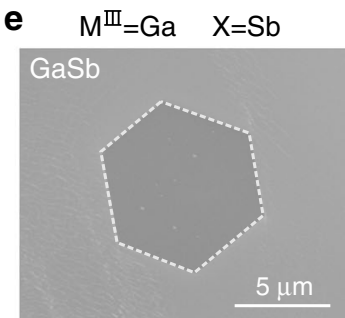

Fig. 2 Universality of the LBL growth toward ultra-thin III-V single crystals. a The schematic diagram of the growth process. b-f SEM images of the synthesized InP, InSb, GaP, GaSb, and GaN single crystals, respectively.

$\mathrm{eV}(\mathrm{InP})^{-1}$, where $(\mathrm{InP})^{-1}$ indicates per unit cell of InP crystal. The value is much larger than that on the InP substrate of $1.08 \mathrm{eV}$ $(\operatorname{InP})^{-1}$, while the adhesion energy on the In metal exhibits the weakest value of $0.66 \mathrm{eV}(\mathrm{InP})^{-1}$. Here, the formation of the $\mathrm{AuIn}_{2}$ alloy is confirmed by the X-ray diffraction (XRD) spectra (Supplementary Fig. 3) and the X-ray photoelectron spectra (XPS) (Supplementary Fig. 4). Regarding that the surface energy of the (111) plane of InP is $\sim 0.66 \mathrm{eV}(\operatorname{InP})^{-1}$ in the reported work ${ }^{27}$, the growth of InP on the $\mathrm{AuIn}_{2}$ substrate would prefer to proceed in a $2 \mathrm{D} \mathrm{LBL}$ growth mode as the $\Delta \gamma=-0.19 \mathrm{eV}(\operatorname{InP})^{-1}$ is $<0$. In addition, the $\Delta \gamma$ of the InP-In system was calculated as a positive value of $0.66 \mathrm{eV}(\mathrm{InP})^{-1}$, which indicates a $3 \mathrm{D}$ island growth behavior of the InP crystals and the formation of bulk crystals on the In surface.

The atomic force microscopy (AFM) image of the typical InP crystal grown on the AuIn 2 alloy with high adhesion strength (Fig. 3b) suggests a triangular morphology with a flat surface. The corresponding height profile (inset of Fig. $3 \mathrm{~b}$ ) indicates an ultrathin thickness of $\sim 6.3 \mathrm{~nm}$, confirming the ultra-thin characteristic of the InP crystal grown on the AuIn ${ }_{2}$ alloy. The AFM image of the crystals grown on the In metal is presented in Fig. 3c, where a height value of $\sim 600 \mathrm{~nm}$ is observed. The result signifies a bulk InP crystal grown on the substrate with a weak adhesion strength, thus validating our growth mechanism. 


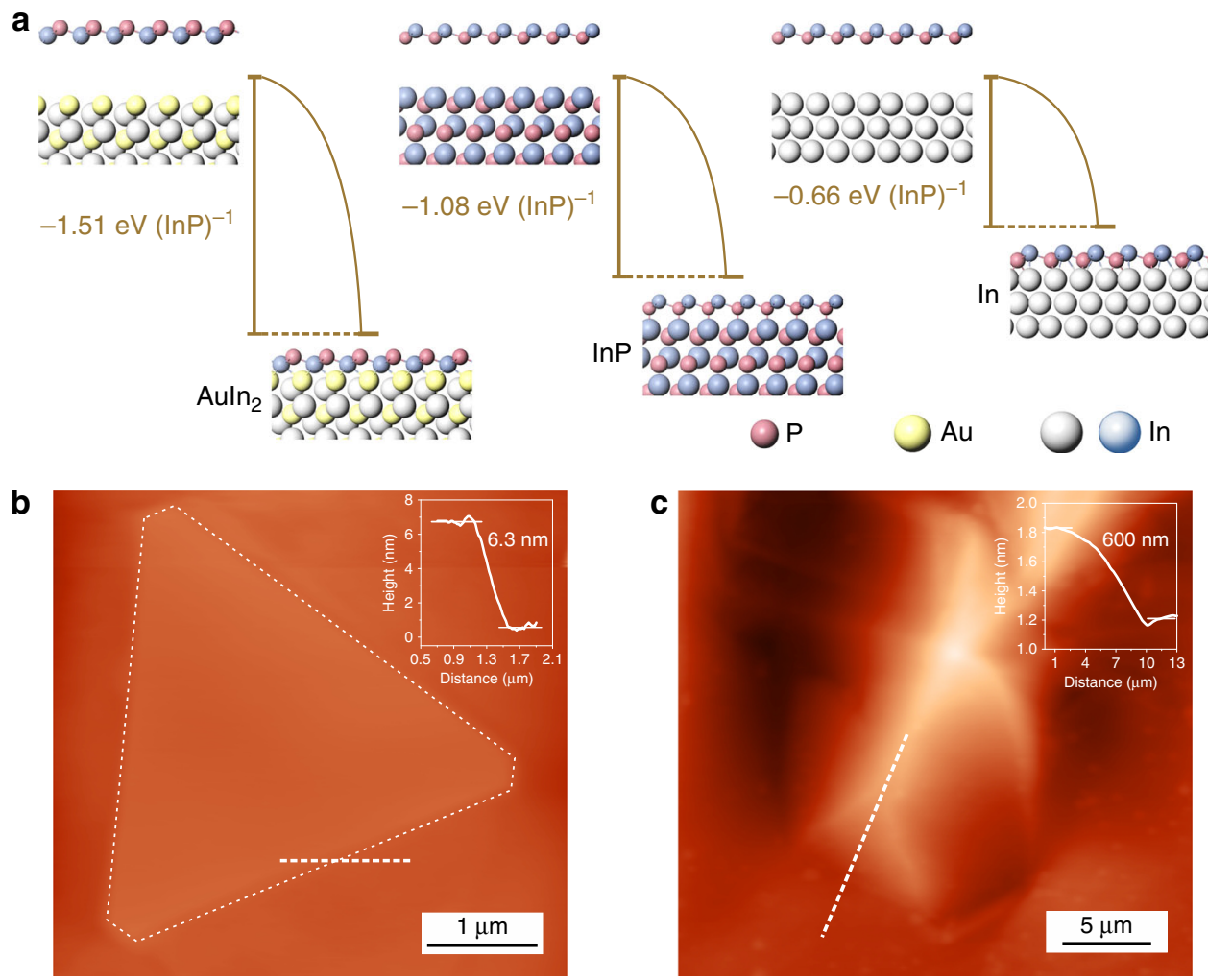

Fig. 3 Theoretical analysis of the growth of $\operatorname{InP}$. a The adhesion energies of InP layers on the surfaces of Auln, InP, and In substrates. $\mathbf{b}$ The AFM image of a typical ultra-thin InP single crystal grown on Auln 2 . The inset shows the corresponding height profile. c The AFM image of a bulk InP crystal grown on In. The inset shows the corresponding height profile.

Here, we believe that the enhanced interaction between the InP layer and $\mathrm{AuIn}_{2}$ substrate is originated from the high adsorption strength of AuIn 2 surface. As shown in Supplementary Fig. 5, the results suggest the stronger adsorption energy of $\mathrm{P}$ atoms on the $\mathrm{AuIn}_{2}$ alloy than that on the InP or the In metal. Furthermore, the universality of the method was also explored to examine the as-proposed growth mechanism. DFT calculations of the adsorption energies of $\mathrm{Sb}$ atom on various substrates were carried out and the results suggest a stronger adsorption strength of $\mathrm{AuIn}_{2}$ alloy compared with that of the In metal and InSb substrates (Supplementary Fig. 6), further proving that the enlarged adsorption strength could enhance the interaction between the substrates and the materials as well as trigger the 2D LBL growth behavior.

Spectral and structural characterizations of crystals. For investigating crystal features of ultra-thin InP single crystals, spectral and structural characterizations were performed. The typical ZB structure of InP crystal and its (111) plane are schemed in Fig. $4 \mathrm{a}$ and $\mathrm{b}$, respectively, which suggest the inherent covalent interaction in the $3 \mathrm{D}$ directions. As the emission wavelength is the most identifiable aspect of a semiconductor with size variation, the photoluminescence $(\mathrm{PL})$ characterization of the ultrathin InP crystal was implemented to investigate the quantum confinement effect (Fig. 4c). The PL emitting peak of ultra-thin InP single crystal at $778 \mathrm{~nm}$ reveals a remarkable blue shift compared with that of the bulk counterparts $(919 \mathrm{~nm})$ shown in Supplementary Fig. 7. This easy observation of quantum confinement effect in ultra-thin InP single crystal may result from its large exciton Bohr radius $(\sim 10 \mathrm{~nm})^{28}$. In addition, the homogeneous color of the PL mapping (Fig. 4d) image suggests the uniform crystallinity of the InP.
To further confirm the atomic structures of InP single crystals, transmission electron microscopy (TEM) characterization was carried out. The corresponding energy-dispersive X-ray spectroscopic (EDS) mapping images of the crystal suggest a good uniformity of the In and P elements (Fig. 4e, f). The collected selected area electron diffraction (SAED) pattern shows only one set of diffraction spots (Fig. 4g), which confirmed the single-crystal nature of the as-grown InP crystal. The EDS spectrum (Fig. 4h) extracted from the InP crystal indicates that the crystal consists of In and $\mathrm{P}$ with a stoichiometric ratio of $\sim 1: 1$. To further analyze the atomic structure, the high-angle annular dark-field scanning transmission electron microscopy (HAADF-STEM) (Fig. 4i) and the bright-field STEM (BF-STEM) (Fig. 4j) images of the InP single crystal are acquired. The ordered atom sequence of In and P is in good agreement with the structure of (111) plane. The lattice fringes recorded from the STEM image exhibit a distance of $0.221 \mathrm{~nm}$, which corresponds to the (220) interplane spacing of the ZB InP structure. Notably, this acquired interplane spacing is larger than that of the bulk counterpart $(0.207 \mathrm{~nm}$, recorded from JPCDS card No. 32-0452), further demonstrating the presence of the increased lattice constant of InP crystal structure. This behavior may be attributed to the change of orbital hybridization induced by the decreased thickness along the $c$ axis for the III-V semiconductors ${ }^{10,11}$. Moreover, the time of flight secondary ion mass spectrometry (TOF-SIMS) was employed to exclude the doping of transition elements (Supplementary Fig. 8). Apart from the InP, the InSb single crystals also exhibit high crystallinity and enlarged lattice parameters, as validated by the TEM characterizations (Supplementary Fig. 9).

Nonlinear optical properties of ultra-thin InP crystals. Considering that the strongly polarized light emission favoring the nonlinear optics of the III-V semiconductors may occur in the $2 \mathrm{D}$ limit according to the predictions ${ }^{13}$, the SHG investigation 
a
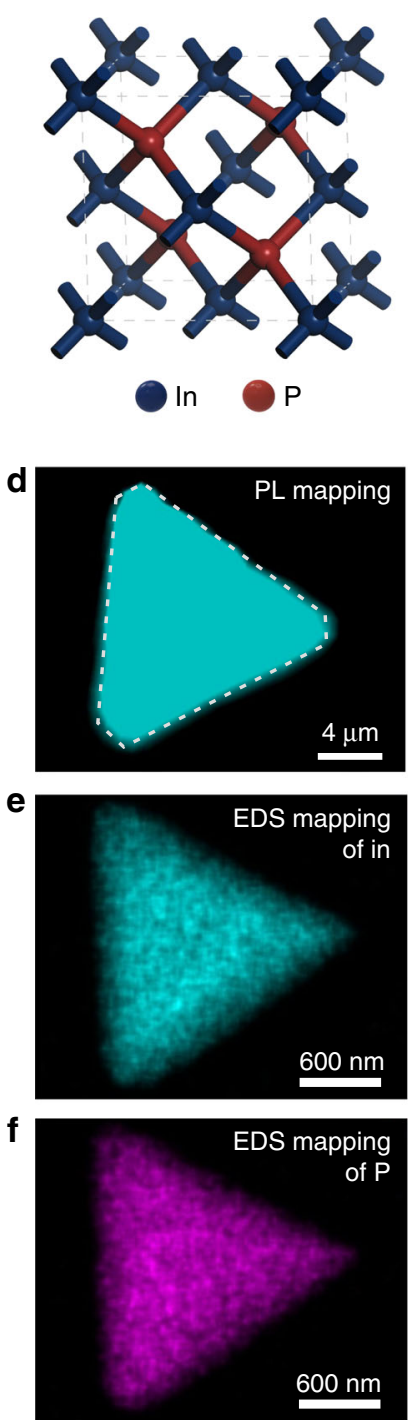

b

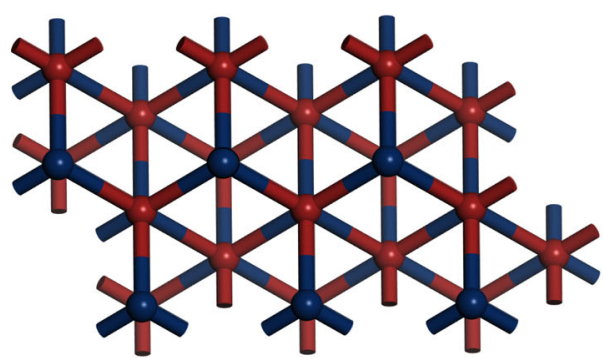

(111) plane
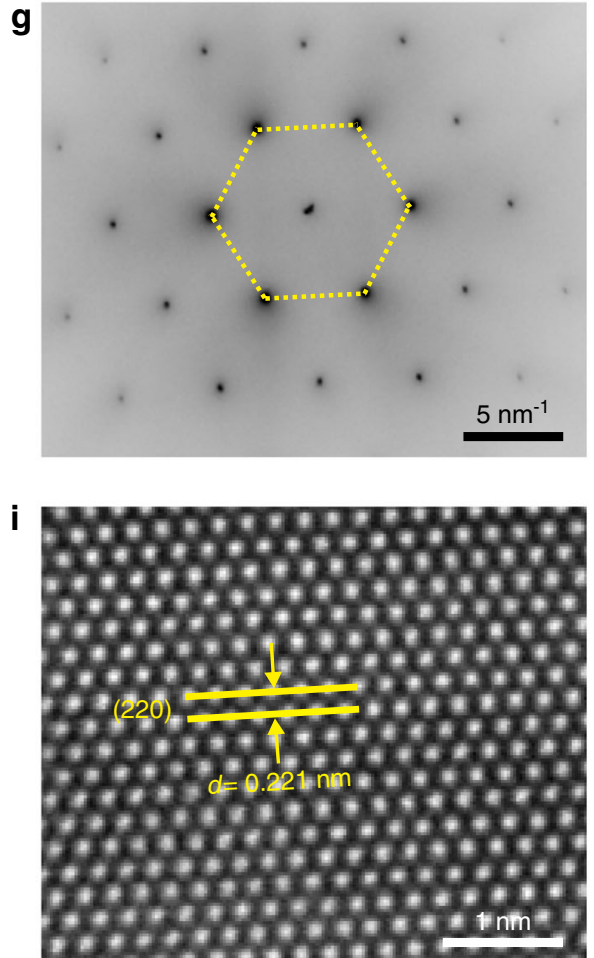
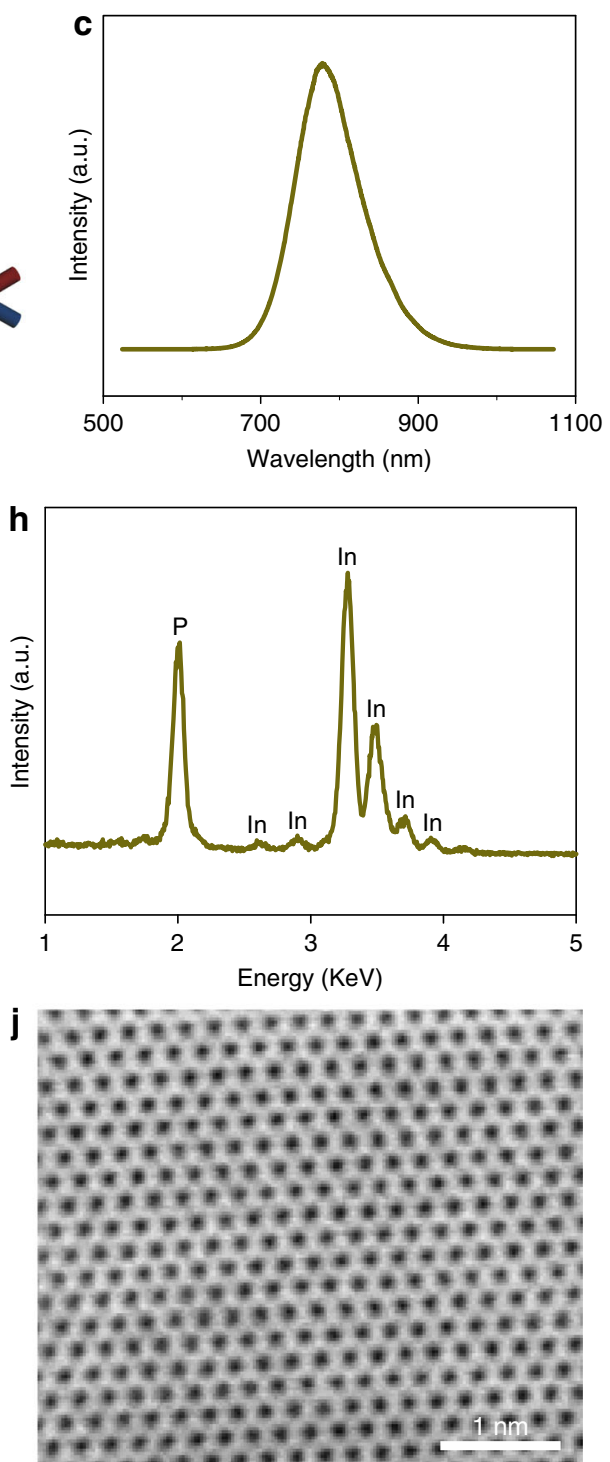

Fig. 4 Spectral and structural characterizations of the ultra-thin InP single crystals. a Scheme of the typical ZB structure of InP crystal. $\mathbf{b}$ Scheme for the (111) plane of ZB InP. c, d The PL spectrum and the corresponding PL mapping of the InP single crystal, respectively. e, $\mathbf{f}$ The EDS mapping of In and P, respectively. $\mathbf{g}$ The SAED patterns of the InP crystal. $\mathbf{h}$ The recorded EDS spectrum of InP. $\mathbf{i}, \mathbf{j}$ The HAADF-STEM and BF-STEM images of the InP crystal acquired along the [111] zone axis.

(Fig. 5a) of the ultra-thin InP single crystals was performed to explore their nonlinear optical properties.

Here, the highest SHG intensity of the ultra-thin InP was recorded at an incident wavelength of $840 \mathrm{~nm}$ femtosecond laser and the output power was fixed at $15 \mathrm{~mW}$, as shown in Fig. $5 \mathrm{~b}$. To probe the power-dependent SHG intensity of InP, the laser power was tuned from 2.8 to $14.8 \mathrm{~mW}$ in $1 \mathrm{~s}$ under the excitation wavelength $\left(\lambda_{\text {excition }}\right)$ of $840 \mathrm{~nm}$. As shown in Fig. $5 \mathrm{c}$, with the increase of the power, the SHG intensity exhibits an evident increase at the peak of $420 \mathrm{~nm}$. The relationship between the laser power and SHG intensity is fitted linearly in a double logarithmic coordinate system, leading to a slope value of 1.85 (Fig. 5d), which approaches the theoretical value of 2 extracted from the electric dipole approximation ${ }^{29}$. The direct observation of strong SHG intensity demonstrates the potential of ultra-thin InP crystal in nonlinear optics. The typical SHG mapping presented in Fig. 5e indicates a uniform SHG intensity, further demonstrating its pure phase. Since the polarization-resolved SHG signal is sensitive to lattice symmetry, the angle-dependent SHG intensity of the InP single crystals was measured. The polarization-resolved
SHG intensity can be fitted according to the formula of $I_{\mathrm{SHG}}=$ $I_{0} \cos ^{2}(3 \theta)$, as shown in Fig. 5f, which exhibits a six-fold anisotropic behavior. It is believed to be induced by the magnified asymmetric structure ${ }^{30,31}$ of the InP with staggered stacking orientation. Notably, the ultra-thin InSb single crystal exhibits a giant SHG response (Supplementary Fig. 10) with the $1 \mathrm{~s}$ exciton $(1410 \mathrm{~nm})$ at a small laser power $(2.05 \mathrm{~mW})$, indicating the extraordinary nonlinear optical properties of ultra-thin InSb.

\section{Discussion}

In summary, we demonstrate that the material-substrate interaction plays a key role in the growth of the ultra-thin III-V crystals because these non-layered materials possess intrinsic covalent bonding in the $3 \mathrm{D}$ directions. By constructing the appropriate alloy substrate with enhanced adhesion strength, the growth of III-V crystals can proceed in the 2D LBL growth mode and various representative ultra-thin III-V single crystals can be successfully obtained. We discover that these highly-crystalline ultrathin III-V systems exhibit remarkable quantum confinement 
a
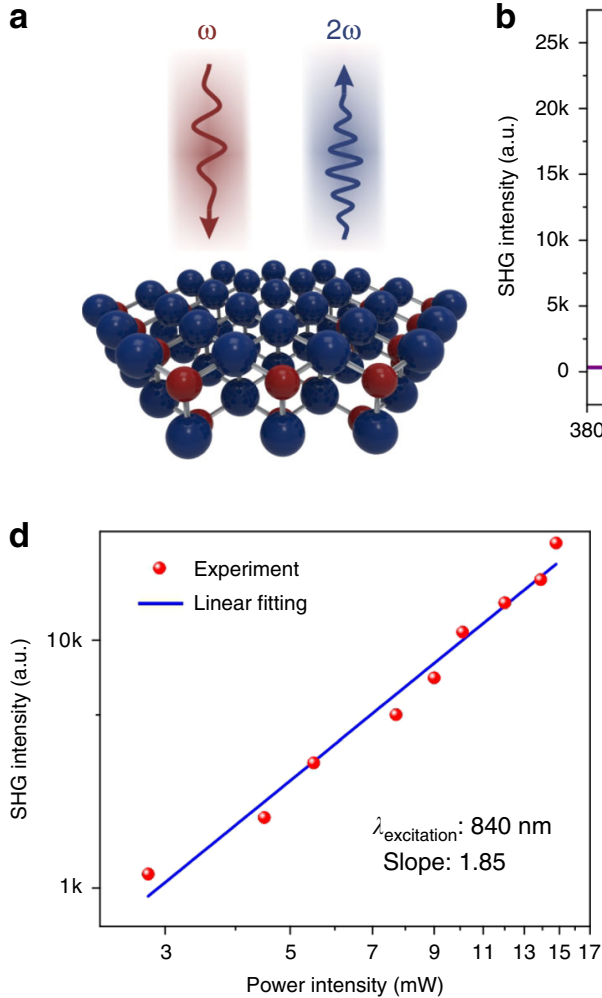

b

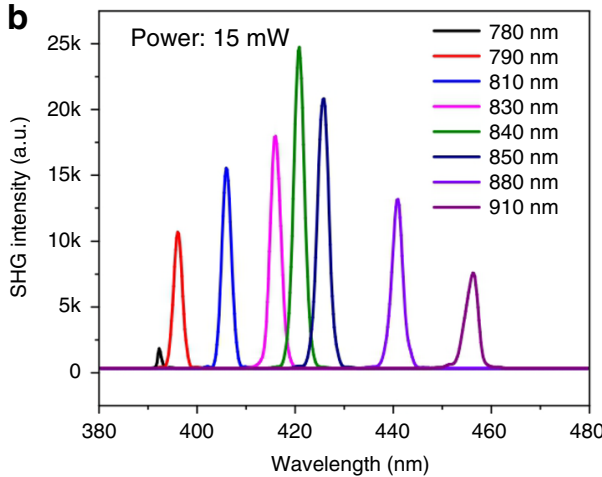

C

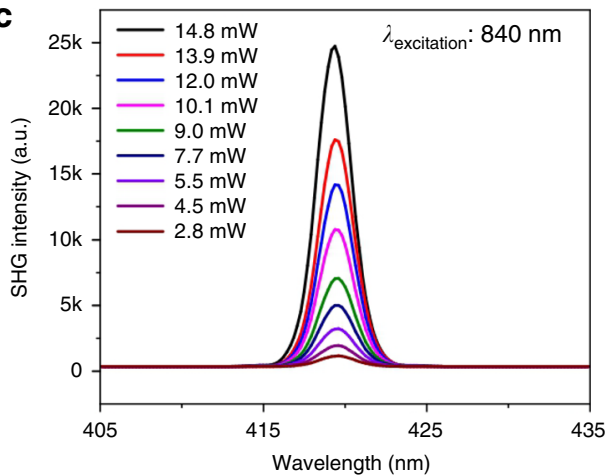

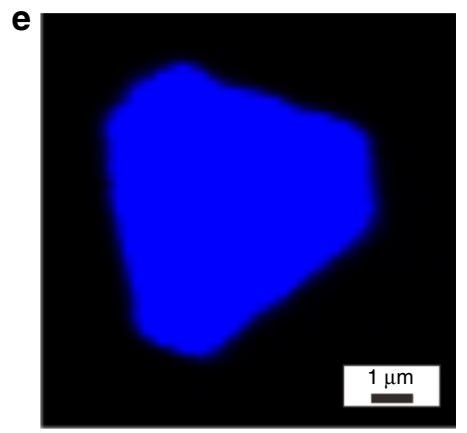

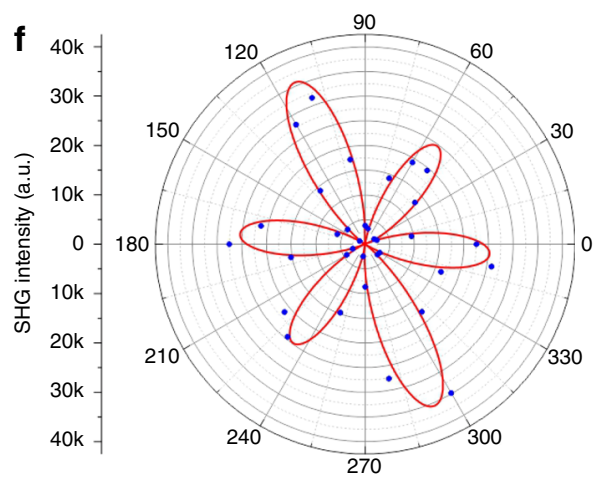

Fig. 5 Nonlinear optical properties of the ultra-thin InP single crystal. a Schematic of the SHG test. b Wavelength-dependent SHG intensity under excitation wavelength from 780 to $910 \mathrm{~nm}$. c Excitation power dependence of SHG intensity. d The linear fitting of power-dependent SHG intensity in logarithmic coordinates. e A SHG mapping of a typical InP single crystal. $\mathbf{f}$ The polarization-resolved SHG signal of the ultra-thin InP crystal.

effect and outstanding nonlinear optical properties. The asachieved breakthrough in the universal synthesis of ultra-thin III-V crystals will greatly promote this material family to win their highlight in the future ultra-thin semiconductor electronics. Our strategy brings forth horizons for realizing the $2 \mathrm{D}$ anisotropic growth of the materials, especially for the non-layered systems.

\section{Methods}

LBL growth process of ultra-thin III-V semiconductors. The growth of ultra-thin III-V single crystals was performed in a quartz tube furnace under ambient pressure. The growth process of ultra-thin InP consists of four steps: (1) Placing the Au wire ( $\sim 5 \mathrm{mg}, 99.999 \%$, China New Metal) and In grain ( $6 \mathrm{mg}, 99.99 \%$, Alfa Aesar) on the W foil $(1 \mathrm{~cm} \times 1 \mathrm{~cm}, 99.95 \%$, Alfa Aesar) in the center region of the quartz tube; (2) Heating the Au-In-W substrate to $1070{ }^{\circ} \mathrm{C}$ under the flow of 300 $\mathrm{sccm} \mathrm{Ar}$ and $300 \mathrm{sccm} \mathrm{H}$ for $5 \mathrm{~min}$; (3) Cooling the $\mathrm{AuIn}_{2}$ alloy substrate to $700^{\circ}$ $\mathrm{C}$ at a rate of $30^{\circ} \mathrm{C} \mathrm{min}^{-1}$ under the flow of Ar and $\mathrm{H}_{2}$ to form $\mathrm{AuIn}_{2}$ alloy; (4) The $\mathrm{AuIn}_{2}$ alloy serving as growth substrate to allow the formation of ultra-thin InP single crystals after heating the P powders $\left(\sim 0.5 \mathrm{mg}, 99.999 \%\right.$, Alfa Aesar) at $200^{\circ} \mathrm{C}$ for 15-25 min. While in the growth process of ultra-thin InSb, the crystals were synthesized on the AuIn 2 alloy at $700^{\circ} \mathrm{C}$ when heating the Sb powders $(\sim 0.5 \mathrm{mg}$, $99.5 \%$, Alfa Aesar) at $650^{\circ} \mathrm{C}$ for $15-20 \mathrm{~min}$. AuGa alloy was fabricated by $\sim 5 \mathrm{mg}$ $\mathrm{Au}$ wire and $\sim 1.8 \mathrm{mg}$ Ga grains $\left(99.99 \%\right.$, Alfa Aesar) via heating at $1070{ }^{\circ} \mathrm{C}$ for 5 $\mathrm{min}$. The ultra-thin $\mathrm{GaP}$ single crystals were grown at $600^{\circ} \mathrm{C}$ with the $\mathrm{P}$ powders $(\sim 0.5 \mathrm{mg})$ heated at $200^{\circ} \mathrm{C}$ for $15-20 \mathrm{~min}$. For the growth process of ultra-thin GaSb single crystals, AuGa alloy was heated at $700{ }^{\circ} \mathrm{C}$ when heating the $\mathrm{Sb}$ powders $(\sim 0.5 \mathrm{mg})$ at $650^{\circ} \mathrm{C}$ for $15-20 \mathrm{~min}$. For the growth process of ultra-thin $\mathrm{GaN}$ single crystals, AuGa alloy was heated at $1000^{\circ} \mathrm{C}$ when heating the $\left(\mathrm{NH}_{2}\right)_{2} \mathrm{CO}$ powders $\left(\sim 5 \mathrm{mg}, 99.5 \%\right.$, Aladdin) at $200^{\circ} \mathrm{C}$ for $5-15 \mathrm{~min}$

Transferring process of the ultra-thin crystals. The transferring process consists of five steps: (1) Spin-coating the poly(methyl methacrylate) (PMMA) films onto the AuIn ${ }_{2}$ substrates with the InP or InSb crystals grown on them; (2) Etching out $\mathrm{AuIn}_{2}$ substrates with the diluted hydrogen chloride (volume ratio of 1:6) for 6-8 $\mathrm{h}$. Consequently, the PMMA/sample films were then released; (3) Removing the metal ions by rinsing with deionized water; (4) Transferring PMMA/sample films onto the target substrates, e.g., $\mathrm{Si}_{/} \mathrm{SiO}_{2}$ substrates and $\mathrm{Cu}$ grids; (5) Dissolving the PMMA layers with hot acetone for obtaining the samples for the subsequent characterization.
Characterizations. The XRD characterization was conducted with a Rigaku Miniflex600. Raman and PL spectra were performed with a laser micro-Raman spectrometer (Renishaw in Via, $532 \mathrm{~nm}$ excitation wavelength). SEM images were obtained on a ZEISS Merlin Compact SEM. The AFM images were taken with an NT-MDT Ntegra Spectra. The XPS measurements were carried out using a Thermo Scientific, ESCALAB 250Xi. The XPS depth analysis was performed at $3 \mathrm{keV}$ with a spot size of $2 \mathrm{~mm} \times 2 \mathrm{~mm}$ and the binding energies were calibrated by referencing the $\mathrm{C} 1 \mathrm{~s}$ peak $(284.8 \mathrm{eV})$. The TEM images were obtained using an aberration-corrected transmission electron microscope (FEI Titan Thermis 60-300) operated at $300 \mathrm{kV}$. The STEM images were obtained using a probe Cs-corrected transmission electron microscope (FEI Titan ChemiSTEM) operating at $200 \mathrm{kV}$. The positive ion TOF-SIMS spectra were acquired with an ION TOF ToF SIMS 5-100.

SHG measurements. The power-dependent SHG measurements of InP crystals were conducted with a Chameleon Ti:Sapphire laser (pulse width $80 \mathrm{fs}$, repetition frequency $80 \mathrm{MHz}$ ). The SHG signal was collected by the WITec alpha 300RS+ Raman system with a $\times 100$ objective $(\mathrm{NA}=0.9)$. The SHG measurements of InSb crystals were conducted with supercontinuum source, SC-PRO-7, YSL. The SHG signal was collected by the Action SpectraPro SP-2300 (300 mm Triple Grating Imaging Spectrometer) with a $\times 100$ objective $(\mathrm{NA}=0.7)$.

DFT calculations. The DFT calculations of the adsorption energies of atoms were carried out using the CASTEP ${ }^{32}$ module in the Materials Studio software (Bio Accelrys). Perdew-Burke-Ernzerhof (PBE) within the generalized gradient approximation (GGA) function ${ }^{33}$ was used for the treatments of electron exchange and correlation energy. The single atoms of $\mathrm{P}$ and Sb were placed in a $20 \times 20 \times 20 \AA^{3}$ cubic cell for calculating the single point energy values, where the energy cutoffs were set to be $320 \mathrm{eV}$ for the single atoms. The geometry optimization of all the systems was performed with a vacuum space of $20 \AA$. The self-consistent-field (SCF) convergence criterions were adopted to be smaller than $10^{-6} \mathrm{eV}$ per atom in the calculation. The adsorption energy $\left(E_{\text {ads }}\right)$ of the $\mathrm{P}$ atom on the $\mathrm{AuIn}_{2}$ layer was calculated based on the equation of $E_{\mathrm{ads}}=E_{\mathrm{P}-\mathrm{AuIn}_{2}}-E_{\mathrm{P}}-E_{\mathrm{AuIn}_{2}}$, where $E_{\mathrm{P}-\mathrm{AuIn}}$, was the energy of P-involved AuIn 2 system, $E_{\mathrm{P}}$ was the energy of the $\mathrm{P}$ atom and $E_{\mathrm{AuIn}_{2}}$ was the energy of the $\mathrm{AuIn}_{2}$ layer. The values of $E_{\text {ads }}$ of other systems were calculated based on the similar equation. The interactions between InP layers and substrates were calculated by the first-principles method with the Vienna ab initio Simulation Package (VASP) ${ }^{34,35}$. The exchange-correlation interaction is GGA-PBE functional and the projector-augmented wave (PAW) technology is used to describe the core electrons ${ }^{36}$. The kinetic energy cutoff for plane-wave basis is $350 \mathrm{eV}$. The relaxation of the electronic degrees of freedom is stopped when the total energy difference is below 
$10^{-5} \mathrm{eV}$ per unit in self-consistent loop, and the structure relaxation is stopped until the max force between atoms is lower than $0.02 \mathrm{eV}^{-1}$. The Grimme-D3(BJ) correction $^{37,38}$ is used to describe the interaction between the InP layer and substrates.

\section{Data availability}

The data that support the findings of this study are available from the corresponding author upon reasonable request.

Received: 16 October 2019; Accepted: 9 July 2020;

Published online: 07 August 2020

\section{References}

1. del Alamo, J. A. Nanometre-scale electronics with III-V compound semiconductors. Nature 479, 317-323 (2011).

2. Brennan, K. \& Hess, K. High field transport in GaAs, InP and InAs. Solid State Electron 27, 347-357 (1984)

3. Wang, J., Gudiksen, M. S., Duan, X., Cui, Y. \& Lieber, C. M. Highly polarized photoluminescence and photodetection from single indium phosphide nanowires. Science 293, 1455-1457 (2001).

4. Kim, Y. et al. Remote epitaxy through graphene enables two-dimensional material-based layer transfer. Nature 544, 340-343 (2017).

5. Wallentin, J. et al. InP nanowire array solar cells achieving $13.8 \%$ efficiency by exceeding the ray optics limit. Science 339, 1057-1060 (2013).

6. Zhang, G. et al. Telecom-band lasing in single InP/InAs heterostructure nanowires at room temperature. Sci. Adv. 5, eaat8896 (2019).

7. Kobayashi, Y., Kumakura, K., Akasaka, T. \& Makimoto, T. Layered boron nitride as a release layer for mechanical transfer of GaN-based devices. Nature 484, 223-227 (2012).

8. Ambacher, O. et al. Two-dimensional electron gases induced by spontaneous and piezoelectric polarization charges in N- and Ga-face $\mathrm{AlGaN} / \mathrm{GaN}$ heterostructures. J. Appl. Phys. 85, 3222-3233 (1999).

9. Kolobov, A. V., Fons, P., Tominaga, J., Hyot, B. \& André, B. Instability and spontaneous reconstruction of few-monolayer thick $\mathrm{GaN}$ graphitic structures. Nano Lett. 16, 4849-4856 (2016).

10. Qin, Z., Qin, G., Zuo, X., Xiong, Z. \& Hu, M. Orbitally driven low thermal conductivity of monolayer gallium nitride $(\mathrm{GaN})$ with planar honeycomb structure: a comparative study. Nanoscale 9, 4295-4309 (2017).

11. Chen, Y. et al. Growth of $2 \mathrm{D}$ GaN single crystals on liquid metals. J. Am. Chem. Soc. 140, 16392-16395 (2018)

12. Al Balushi, Z. Y. et al. Two-dimensional gallium nitride realized via graphene encapsulation. Nat. Mater. 15, 1166-1171 (2016).

13. Sanders, N., Bayerl, D., Shi, G., Mengle, K. A. \& Kioupakis, E. Electronic and optical properties of two-dimensional GaN from first-principles. Nano Lett. 17, 7345-7349 (2017).

14. Chen, Y. et al. GaN in different dimensionalities: properties, synthesis, and applications. Mater. Sci. Eng. R. 138, 60-84 (2019).

15. Duan, X. \& Lieber, C. M. General synthesis of compound semiconductor nanowires. Adv. Mater. 12, 298-302 (2000).

16. Kitauchi, Y. et al. Structural transition in indium phosphide nanowires. Nano Lett. 10, 1699-1703 (2010)

17. Waltereit, P. et al. Nitride semiconductors free of electrostatic fields for efficient white light-emitting diodes. Nature 406, 865-868 (2000).

18. Liu, B. et al. Template approach to crystalline GaN nanosheets. Nano Lett. 17, 3195-3201 (2017).

19. Wu, J., Zhang, D., Lu, Q., Gutierrez, H. R. \& Eklund, P. C. Polarized Raman scattering from single GaP nanowires. Phys. Rev. B 81, 165415 (2010).

20. Yang, C. C. \& Li, S. Size-dependent raman red shifts of semiconductor nanocrystals. J. Phys. Chem. B 112, 14193-14197 (2008).

21. Adu, K. W., Gutiérrez, H. R., Kim, U. J., Sumanasekera, G. U. \& Eklund, P. C. Confined phonons in Si nanowires. Nano Lett. 5, 409-414 (2005).

22. Yang, M. et al. Study of the Raman peak shift and the linewidth of lightemitting porous silicon. J. Appl. Phys. 75, 651-653 (1994).

23. Aoki, K., Anastassakis, E. \& Cardona, M. Dependence of Raman frequencies and scattering intensities on pressure in GaSb, InAs, and InSb semiconductors. Phys. Rev. B 30, 681-687 (1984).

24. Sanatinia, R., Swillo, M. \& Anand, S. Surface second-harmonic generation from vertical GaP nanopillars. Nano Lett. 12, 820-826 (2012).

25. Dick, K. A. A review of nanowire growth promoted by alloys and non-alloying elements with emphasis on Au-assisted III-V nanowires. Prog. Cryst. Growth Charact. Mater. 54, 138-173 (2008).

26. Mutaftschiev, B. Wetting and displacement of three-dimensional and twodimensional layers on a foreign substrate. Phys. Rev. B 40, 779 (1989).
27. Liu, Q., Moll, N., Scheffler, M. \& Pehlke, E. Equilibrium shapes and energies of coherent strained InP islands. Phys. Rev. B 60, 17008 (1999).

28. Giessen, H. et al. Observation of the quantum confined ground state in InP quantum dots at $300 \mathrm{~K}$. Appl. Phys. Lett. 68, 304-306 (1996).

29. Shimizu, A. Optical nonlinearity induced by giant dipole moment of wannier excitons. Phys. Rev. Lett. 61, 613-616 (1988).

30. Gong, C. et al. Self-confined growth of ultrathin 2D nonlayered wide-bandgap semiconductor CuBr flakes. Adv. Mater. 31, 1903580 (2019).

31. Jiang, T. et al. Valley and band structure engineering of folded $\mathrm{MoS}_{2}$ bilayers. Nat. Nanotechnol. 9, 825-829 (2014).

32. Clark, S. J. et al. First principles methods using CASTEP. Z. Kristallogr 220, 567 (2005)

33. Perdew, J. P., Burke, K. \& Ernzerhof, M. Generalized gradient approximation made simple. Phys. Rev. Lett. 77, 3865-3868 (1996).

34. Kresse, G. \& Furthmuller, J. Efficient iterative schemes for ab initio totalenergy calculations using a plane-wave basis set. Phys. Rev. B 54, 11169-11186 (1996).

35. Kresse, G. \& Furthmüller, J. Efficiency of ab-initio total energy calculations for metals and semiconductors using a plane-wave basis set. Comp. Mater. Sci. 6 , 15-50 (1996).

36. Blochl, P. E. Projector augmented-wave method. Phys. Rev. B 50, 17953-17979 (1994).

37. Grimme, S., Antony, J., Ehrlich, S. \& Krieg, H. A consistent and accurate ab initio parametrization of density functional dispersion correction (DFT-D) for the 94 elements H-Pu. J. Chem. Phys. 132, 154104 (2010).

38. Grimme, S., Ehrlich, S. \& Goerigk, L. Effect of the damping function in dispersion corrected density functional theory. J. Comput. Chem. 32, 1456-1465 (2011)

\section{Acknowledgements}

The research was supported by the National Natural Science Foundation of China (Grants No. 21673161, 21905210, 51728202), and the Sino-German Center for Research Promotion (Grant No. 1400). L.F. acknowledges support by Wuhan University President's Funding.

\section{Author contributions}

L.F. developed the concept and conceived the experiments. Y.X.C. and J.X.L. carried out the main experiments and wrote the paper. L.F. and M.Q.Z. revised the paper. L.F., Y.X. C., J.X.L., M.Q.Z., F.Y.L., T.R.L., Y.C., H.H.L., B.W., R.S., J.F.G., and Z.C.W. contributed to the data analysis and scientific discussion.

\section{Competing interests}

The authors declare no competing interests.

\section{Additional information}

Supplementary information is available for this paper at https://doi.org/10.1038/s41467020-17693-5.

Correspondence and requests for materials should be addressed to L.F.

Peer review information Nature Communications thanks Joel Ager III and the other, anonymous reviewer(s) for their contribution to the peer review of this work.

Reprints and permission information is available at http://www.nature.com/reprints

Publisher's note Springer Nature remains neutral with regard to jurisdictional claims in published maps and institutional affiliations.

Open Access This article is licensed under a Creative Commons Attribution 4.0 International License, which permits use, sharing, adaptation, distribution and reproduction in any medium or format, as long as you give appropriate credit to the original author(s) and the source, provide a link to the Creative Commons license, and indicate if changes were made. The images or other third party material in this article are included in the article's Creative Commons license, unless indicated otherwise in a credit line to the material. If material is not included in the article's Creative Commons license and your intended use is not permitted by statutory regulation or exceeds the permitted use, you will need to obtain permission directly from the copyright holder. To view a copy of this license, visit http://creativecommons.org/ licenses/by/4.0/.

(C) The Author(s) 2020 